\title{
THEORY AND PRACTICAL ATTAINMENTS IN THE DESIGN AND USE OF RADIO-DIRECTION FINDING APPARATUS USING CLOSED COIL ANTENNAS.*
}

BY

\author{
A. S. BLATTERMAN. \\ Captain Signal Corps, U. S. Army. \\ (SUPPLEMENTARY NOTE.)
}

INFORMATION concerning coil receivers for wave-lengths below Iooo metres has already appeared in this JoURNAL ${ }^{1}$ in connection with the data for longer wave-lengths. The following results on $4^{\prime}$ and $6^{\prime}$ loops for $1 / 4^{\prime \prime}$ and $7 / 10^{\prime \prime}$ wire spacing, respectively, which values correspond to the best values of spacing for these sizes of loops as determined by the curve, Fig. 2I, page 309. of the original paper, have recently been obtained:

\begin{tabular}{|c|c|c|c|c|c|}
\hline \multicolumn{2}{|c|}{ Four turns. } & \multicolumn{2}{|c|}{ Seven turns. } & \multicolumn{2}{|c|}{ Ten turns. } \\
\hline Wave-length. & Resistancé. & Wave-length. & Resistance. & Wave-length. & Resistance. \\
\hline 220 metres & I 4.8 ohms & 330 metres & 10.5 ohms & $45^{\circ}$ metres & I3.2 ohms \\
\hline 275 metres & 3.95 ohms & 390 metres & 7.5 ohms & 600 metres & 6.8 ohms \\
\hline $35^{\circ}$ metres & 2.65 ohms & $45^{\circ}$ metres & 5.55 ohms & 800 metres & 4.55 ohms \\
\hline $45^{\circ}$ metres & 2.15 ohms & 600 metres & 3.6 ohms & Iooo metres & 3.6 ohms \\
\hline 600 metres & 1.6 ohms & Iooo metres & 2.5 ohms & I 400 metres & 2.8 ohms \\
\hline 800 metres & I.4 ohms & & & & \\
\hline
\end{tabular}

\begin{tabular}{|c|c|c|c|c|c|}
\hline \multicolumn{6}{|c|}{ Six-foot Loop- $\frac{7 \overline{1} \bar{\prime}}{}$ 'Spacing. } \\
\hline \multicolumn{2}{|c|}{ Three turns. } & \multicolumn{2}{|c|}{ Five turns. } & \multicolumn{2}{|c|}{ Seven turns. } \\
\hline I 80 metres & I I.4 ohms & 275 metres & $20.0 \mathrm{ohms}$ & 400 metres & $20.0 \mathrm{ohms}$ \\
\hline 260 metres & 5.5 ohms & 305 metres & I $2.9 \mathrm{ohms}$ & $55^{\circ}$ metres & 8.6 ohms \\
\hline 360 metres & 3.4 ohms & 400 metres & $6.5 \mathrm{ohms}$ & 750 metres & 6.0 ohms \\
\hline 500 metres & 2.5 ohms & 500 metres & $4.6 \mathrm{ohms}$ & 1200 metres & $3 \cdot 4$ ohms \\
\hline 750 metres & 2.0 ohms & $75^{\circ}$ metres & 3.0 ohms & & \\
\hline 1000 metres & I.9 ohms & & & & \\
\hline
\end{tabular}

Ten Turns.

$\begin{array}{rr}660 \text { metres } & 22.6 \mathrm{ohms} \\ 600 \text { metres } & \text { I7.2 ohms } \\ 750 \text { metres } & \text { I0.5 ohms } \\ \text { I000 metres } & 6.5 \mathrm{ohms} \\ \text { I 500 metres } & 4.4 \mathrm{ohms}\end{array}$

* Communicated by Maj.-Gen. George Owen Squier, Chief Signal Officer, U. S. A.

${ }^{1}$ Vol. 188, page 289 (September, I9I9).

VoL. I90, No. I137-30 